\title{
Etiology and surgical treatment of pediatric trigger finger: Quantitative measurements of the cross- sectional configuration of the flexor tendon using ultrasonography
}

Jihyeung Kim ( $\square$ kjh12344@hanmail.net)

Seoul National University

Qingyuan Li

Tianjin Hospital

Linying Cao

Seoul National University

Goo Hyun Baek

Seoul National University

Haihua Zhan

Tianjin Hospital

\section{Research Article}

Keywords: Pediatric trigger finger, Ultrasonography, Surgical treatment

Posted Date: February 4th, 2022

DOI: https://doi.org/10.21203/rs.3.rs-1319969/v1

License: (c) (i) This work is licensed under a Creative Commons Attribution 4.0 International License.

Read Full License 


\section{Abstract}

Background: This study quantitively evaluated the cross-sectional area of the flexor tendon and the area under the A1 pulley using ultrasonography in patients with unilateral pediatric trigger finger (PTF). The surgical outcome following release of the A1 pulley together with the proximal one-third of the A2 pulley was determined.

Methods: We enrolled 32 trigger fingers of 29 patients diagnosed with unilateral PTF. The cross-sectional configuration of the flexor tendon was determined in 12 trigger fingers of 10 patients using ultrasonography. The ultrasonography measurements consisted of the anteroposterior (AP) diameter, radioulnar $(\mathrm{RU})$ diameter, cross-sectional area of the flexor tendon at the level of the greatest AP diameter of the flexor tendon proximal to the A1 pulley, and the inner dimensions of the A1 pulley. The measurements were repeated on the contralateral normal same finger. The efficacy of the surgical release of the A1 pulley together with that of the proximal one-third of the A2 pulley was assessed in 11 trigger fingers of 11 patients who underwent surgical intervention for PTF.

Results: The average RU measurement and cross-sectional area of the flexor tendon were larger than the inner dimensions of the A1 pulley in PTF patients. The average AP, RU, and cross-sectional area measurements in the area under the A1 pulley were significantly larger in the trigger finger than in the contralateral normal finger $(P<0.05)$. The average RU and cross-sectional area measurements of the flexor tendon proximal to the A1 pulley were also significantly larger in the trigger finger than in the contralateral normal finger $(P<0.05)$. In 10 of the $11(91 \%)$ surgically treated fingers, successful resolution of triggering without recurrence was achieved after release of the A1 pulley together with the proximal one-third of the A2 pulley. One finger had recurrent triggering, which was successfully treated with complete A2 pulley and partial A3 pulley release. The patient remained asymptomatic after the revision procedure. No other complications were observed. All patients were satisfied with the surgical outcomes and at the most recent follow-up evaluation had returned to full activities.

Conclusions: Ultrasonographic measurements in PTF patients suggested that PTF is caused by a size mismatch between the flexor tendon and the area under the A1 pulley. The difference is the result of nodule formation or thickening within one or both of the flexor digitorum superficialis slips. Surgical release of the A1 pulley and of the proximal one-third of the A2 pulley is a very efficient treatment for PTF, yielding satisfactory outcomes that may shorten the duration of symptoms.

\section{Background}

Pediatric trigger finger (PTF) is one tenth as common as pediatric trigger thumb and differs from adult trigger finger [1]. Although once considered congenital, there are no reports of trigger finger being present at birth. Thus, like trigger thumb, PTF is an acquired, not a congenital condition [2, 3]. PTF typically develops in children between the ages of 3 weeks and 11 years [1]. Patients often present with a 
decreased active range of motion, or triggering, but some present with fixed flexion contracture [4]. The middle finger is most commonly affected although one or more digits may be involved $[1,5]$.

PTF is rare and its etiology remains unknown, although several studies have suggested that flexor tendon abnormalities account for triggering or flexion deformity [1, 5-7]. Nodular thickening or fusiform swelling of the flexor tendon has also been described. The result is likely to be a mismatch in the sizes of the flexor tendon and A1 pulley over the metacarpophalangeal joint, thus disrupting normal tendon gliding, as occurs in pediatric trigger thumb $[3,4,8,9]$. Although published management algorithms and strategies have reported good outcomes with a variety of treatments, there is no consensus regarding the best method to treat PTF $[4,10,11]$. The management of PTF ranges from nonsurgical measures (observation, physical therapy, and splinting) to operative exploration and correction of the offending structures [1, 5 , $12,13]$. In most patients, treatment is initially non-operative but if it fails then surgical intervention is usually recommended. Historically, the results of the surgical management of PTF have been far less predictable than is the case in pediatric trigger thumb and adult trigger finger, with recurrence rates as high as 44\% [1]. Among the surgical methods described in the literature are flexor digitorum superficialis (FDS) decussation for the flexor digitorum profundus (FDP), A1 and partial A2 pulley division, and partial or complete resection of the FDS tendon $[1,5]$.

In this study, patients with unilateral PTF were quantitively evaluated using ultrasonography to determine the cross-sectional area of the flexor tendon and the area under the A1 pulley, to obtain insights into the etiology of PTF. We also present the surgical outcome achieved by the release of the A1 pulley and the proximal one-third of the A2 pulley in patients with PTF.

\section{Methods}

\section{Patients}

We retrospectively reviewed 38 patients diagnosed with PTF who underwent ultrasound at the initial visit to our clinic between January 2007 and December 2020. This study was reviewed and approved by the relevant Institutional Review Board. The inclusion criteria were as follows: diagnosis of unilateral PTF, routinely performed ultrasound of both the affected and the contralateral normal same finger, and followup $>1$ year. Patients with other, associated disorders, such as juvenile idiopathic arthritis or Hurler syndrome and Hunter syndrome, were excluded. Thus, 32 trigger fingers of 29 patients ( 16 females and 13 males; mean age, 46 months [range: 2-135 months]) were evaluated. The right side was affected in 19 patients, and the left was affected in 10. In all, 11 trigger fingers of 11 patients ( 6 females and 5 males; mean age, 56 months [range: 2-135 months]) were treated surgically and 21 trigger fingers of 18 patients (10 female and 8 male patients; mean age, 87 months [range: 7-88]) were not. Of the latter group, six children had a history of triggering but were free of symptoms at the initial examination at our clinic and have had no further problems. Twelve other children had minor triggering without fixed flexion contracture, such that surgery was not indicated. In these fingers spontaneous complete recovery eventually occurred. In addition, among the 29 patients, the cross-sectional configuration of the flexor 
tendon of 12 trigger fingers of 10 patients ( 4 females and 6 males; mean age, 36 months [range, 12-78 months]) was measured using ultrasonography. The average follow-up was 43 (range, 12-144) months.

\section{Ultrasonographic evaluation}

At the initial examination, both the affected and the contralateral normal finger were assessed via ultrasonography. The measurements were performed using a high-frequency ultrasonic transducer (range, 15-8 MHz) and consisted of the anteroposterior (AP) diameter and the radioulnar (RU) diameter of the cross-section of the flexor tendon (defined as the sum of the FDS and FDP at the level of the greatest AP diameter of the flexor tendon proximal to the A1 pulley) (Fig. 1). Data from the two measurements were used to evaluate the conformational changes in the flexor tendon. The crosssectional area of the flexor tendon was calculated from the AP and RU diameters. These parameters were also measured for the inner dimensions of the A1 pulley. Two of the authors measured the relevant parameters twice, using previously acquired ultrasonographic images, at intervals of 2 weeks to determine interobserver and intraobserver reliability [14]. The average of the two measurements was used. The AP and RU measurement ratios were defined as the AP and RU measurements of the flexor tendon divided by the AP and RU measurements of the area under the A1 pulley. Likewise, the area ratio was defined as the cross-sectional area of the flexor tendon divided by the cross-sectional area of the area under the A1 pulley.

\section{Release of the A1 pulley and the proximal one-third of the A2 pulley in patients with PTF}

In the surgical treatment of PTF, a Bruner incision was made such that the area proximal to the A1 pulley to distal to the A2 pully was exposed. The incision was initially made over the A1 pulley (Fig. 2). After the adjacent neurovascular structures had been identified and protected, the A1 pulley was released. This was followed by an intraoperative check of the full excursion of the flexor tendon with its sheath, tested by exerting traction on the FDS and FDP tendons and by passively flexing and extending the digit. If triggering was still present, the incision was extended to expose the A2 pulley. Additional release of the proximal one-third of the A2 pulley was performed (Fig. 3). Full passive motion without triggering was confirmed. Postoperatively, a bulky soft dressing was applied, with early motion initiated after wound healing. The average follow-up was 40 (range, 12-115) months.

\section{Statistical analysis}

A paired t-test was used to compare the AP, RU, and area measurements of the flexor tendon and those of the area under the A1 pulley between the trigger finger and contralateral normal finger. The AP measurement ratio, RU measurement ratio, and the area ratio between the trigger finger and the contralateral normal finger were also compared, using a paired t-test. Intraclass correlation coefficients 
were calculated to assess interobserver and intraobserver reliability. A $P$ value $<0.05$ was considered to indicate statistical significance. All statistical analyses were performed using SPSS for Windows (ver.19.0.1; IBM, Chicago, IL).

\section{Results}

In 32 trigger fingers of 29 patients, the most commonly affected finger was the middle finger (16 fingers, $50 \%$ ), followed by the ring finger ( 12 fingers, $38 \%$ ), the index finger ( 3 fingers, $9 \%$ ), and the little finger ( 1 finger, $3 \%)$.

The cross-sectional RU and area measurements of the flexor tendon and those of the area under the A1 pulley significantly differed between the trigger finger and the contralateral normal finger $(P<0.05)$ (Fig. 4). The cross-sectional AP measurements of the area under the A1 pulley also significantly differed between the trigger finger and the contralateral normal finger $(P<0.05)$, whereas the AP measurements of the flexor tendon of the trigger finger and the contralateral normal finger did not $(P=0.26)$. Both the RU measurement ratio and the area ratio significantly differed between the trigger finger and the contralateral normal finger $(P<0.05)$. Interobserver and intraobserver reliability for the ultrasonographic measurements was excellent (0.98 and 0.99).

In the 11 patients who underwent surgical treatment, the interval from presentation to surgery ranged from 13 days to 46 months (average, 16 months). In all of these patients, nodule formation or thickening within one or both of the FDS slips was determined intraoperatively. In addition, while there was no abnormal relationship between the FDS and FDP tendons, mild to severe tenosynovitis was seen between the FDS and FDP tendons. After release of the A1 pulley and proximal one-third of the A2 pulley, a tenosynovectomy was performed between the FDS and FDP tendons. All 11 trigger fingers except one went on to successful healing, with full return to function and no recurrent triggering or pain at the latest follow-up examination. The one patient with recurrence had a second procedure 1 year after the first, during which the complete A2 pulley and part of the A3 pulley were released. After the revision procedure, the patient had a full return of motion without triggering. There were no other complications.

\section{Discussion}

PTF is much less common than pediatric trigger thumb $[1,5,15]$. Although many investigators have reviewed trigger finger in children, there are few specific reviews that do not include trigger thumb. The exact etiology of PTF is unknown, but nodularity or thickening of the FDS or FDP tendons, an abnormal relationship between the FDS and FDP tendons, proximal FDS decussation, and constriction of the A1 or A2 pulley have been described $[1,5,6,16,17]$.

In this study, among the 12 trigger fingers of 10 patients in which the cross-sectional configuration of the flexor tendon was measured using ultrasonography, we identified an enlargement of the flexor tendon (AP, $\mathrm{RU}$, and area measurements) in the affected trigger finger compared to the contralateral normal finger. 
The RU and area measurements of the flexor tendon proximal to the A1 pulley were increased compared to those of the flexor tendon under the A1 pulley. This mismatch may be a key factor in the etiology of PTF, as it would result in the flexor tendon proximal to the A1 pulley that is too large to fit into the pulley. In addition, during surgery for 11 trigger fingers in 11 patients, a nodule or thickening within one or both of the FDS slips was consistently found intraoperatively. There was no abnormal relationship between the FDS and FDP tendons nor was there a narrowing of either the A1 or A2 pulley itself. We therefore conclude that the size mismatch resulted from the anatomical abnormalities, most commonly a nodule or thickening within one or both of the FDS slips.

Due to the rarity of PTF, there is no consensus in the literature on the best form of treatment. Whether trigger finger resolves spontaneously is unclear. De Smet et al. followed 15 trigger fingers of 10 patients over a 6-year period between 1990 and 1996 [18]. Two patients (20\%) had a spontaneous recovery. Moon et al. reported eight PTFs over a 3-year period between 1995 and 1998, with spontaneous resolution of the triggering symptoms occurring in all eight and no fingers requiring follow-up surgery [2]. In our study, spontaneous complete recovery with no further surgery occurred in 21 of the 32 trigger fingers $(66 \%)$.

The successful surgical management of PTF has been described in several reports. The procedures found to be safe and reliable include widening of the FDS decussation for the FDP, A1 and partial A2 pulley division, and partial, complete, or partial V-shaped resection of the FDS tendon $[1,5,6,11,19]$. In this study, in all 11 surgically patients the procedure consisted of surgical release of the proximal onethird of the A2 pulley in addition to the A1 pulley. Successful healing with a full return of function and no recurrent triggering or pain at the latest follow-up examination was achieved in all but one patient. In that case, recurrent triggering was successfully treated with complete A2 pulley and partial A3 pulley release.

This study had several limitations. First, the number of patients was small and the study was retrospective in its design. Second, patients with bilateral trigger finger were not included. Third, selection bias may have occurred because patients who did not undergo ultrasonographic evaluations were excluded.

\section{Conclusions}

Based on ultrasonographic measurements, PTF may arise from a size mismatch between the flexor tendon and the area under the A1 pulley. The size mismatch may be caused by a nodule or thickening within one or both of the FDS slips. A surgical procedure consisting of the release of the A1 pulley and proximal one-third of the A2 pulley is recommended in PTF patients, as it provides satisfactory outcomes that may shorten the duration of symptoms.

\section{Abbreviations}


PTF: Pediatric trigger finger; AP: Anteroposterior; RU: Radioulnar; FDS: Flexor digitorum superficialis; FDP: Flexor digitorum profundus

\section{References}

1. Cardon LJ, Ezaki M, Carter PR. Trigger finger in children. J Hand Surg Am 1999;24:1156-61

2. Moon WN, Suh SW, Kim IC. Trigger digits in children. J Hand Surg Br 2001;26:11-2

3. Rodgers WB, Waters PM. Incidence of trigger digits in newborns. J Hand Surg Am 1994;19:364-8

4. Shah AS, Bae DS. Management of pediatric trigger thumb and trigger finger. J Am Acad Orthop Surg 2012;20:206-13

5. Tordai P, Engkvist O. Trigger fingers in children. J Hand Surg Am 1999;24:1162-65

6. Bae DS, Sodha S, Waters PM. Surgical treatment of the pediatric trigger finger. J Hand Surg Am 2007;32:1043-7

7. Steenwerckx A, De Smet L, Fabry G. Congenital trigger digit. J Hand Surg Am 1996;21:909-11

8. Paaske BP, Soe-Nielsen NH, Noer HH. Release of trigger finger in children. Long term results. Scand $J$ Plast Reconstr Surg Hand Surg 1995;29:65-7

9. Kim J, Gong HS, Seok HS, et al. Quantitative Measurements of the Cross-sectional Configuration of the Flexor Pollicis Longus Tendon Using Ultrasonography in Patients With Pediatric Trigger Thumb. $J$ Hand Surg Am 2018;43:284 e281-7

10. Giugale JM, Fowler JR. Trigger Finger: Adult and Pediatric Treatment Strategies. Orthop Clin North Am 2015;46:561-9

11. Schaverien MV, Godwin Y. Paediatric trigger finger: Literature review and management algorithm. $J$ Plast Reconstr Aesthet Surg 2011;64:623-31

12. Nemoto $\mathrm{K}$, Nemoto $\mathrm{T}$, Terada $\mathrm{N}$, et al. Splint therapy for trigger thumb and finger in children. $J$ Hand Surg Br1996;21:416-8

13. Tsuyuguchi Y, Tada K, Kawaii H. Splint therapy for trigger finger in children. Arch Phys Med Rehabil 1983;64:75-6

14. Engh CA, Jr., Sychterz CJ, Young AM, et al. Interobserver and intraobserver variability in radiographic assessment of osteolysis. J Arthroplasty 2002;17:752-9

15. Mulpruek P, Prichasuk S, Orapin S. Trigger finger in children. J Pediatr Orthop 1998;18:239-41

16. Hirata $\mathrm{H}$, Fujisawa $\mathrm{K}$, Sasaki $\mathrm{H}$, et al. Congenital triggering of the index finger at the A2 pulley. $J$ Hand Surg Br1996;21:609-11

17. Weilby A. Trigger finger. Incidence in children and adults and the possibility of a predisposition in certain age groups. Acta Orthop Scand 1970;41:419-27

18. De Smet L, Steenwerckx A, Van Ransbeeck $H$. The so-called congenital trigger digit: further experience. Acta Orthop Belg 1998;64:306-8 
19. Hamada Y, Tonogai I, Hibino N, et al. Etiology and treatment of pediatric trigger finger: possible application of partial V-shaped resection of the central part of flexor digitorum superficialis through our case presentation. Hand (N Y) 2011;6:115-6

\section{Figures}

\section{Figure 1}

Ultrasonographic images of a 27-month-old girl with pediatric trigger finger on the right middle digit. $\mathbf{A}$. Transverse image of the flexor tendon proximal to the A1 pulley on the right side. B. Transverse image of the area under the $\mathrm{A} 1$ pulley on the right side. $\mathbf{C}$. Transverse image of the flexor tendon proximal to the A1 pulley on the left side. $\mathbf{D}$. Transverse image of the area under the A1 pulley on the left side.

\section{Figure 2}

Intraoperative photos show a tight A1 pully relative to the proximal flexor tendon.

\section{Figure 3}

Intraoperative photos after release of the A1 pulley and proximal one-third of the A2 pulley.

\section{Figure 4}

Comparisons of the parameters of the trigger finger and contralateral normal finger. The asterisk indicates a significant difference between them. 